\title{
Two sugar beet chitinase genes, BvSP2 and BvSE2, analysed with SNP Amplifluor-like markers, are highly expressed after Fusarium root rot inoculations and field susceptibility trial
}

\author{
Raushan Yerzhebayeva ${ }^{1}$, Alfiya Abekova ${ }^{1}$, Kerimkul Konysbekov ${ }^{2}$, Sholpan Bastaubayeva ${ }^{1}$, Aynur \\ Kabdrakhmanova ${ }^{3}$, Aiman Absattarova ${ }^{4}$, Yuri Shavrukov ${ }^{\text {Corresp. }} 5$ \\ ${ }^{1}$ Kazakh Research Institute of Agriculture and Plant Production, Almalybak, Almaty district, Kazakhstan \\ 2 Taldykorgan branch, Kazakh Research Institute of Agriculture and Plant Production, Taldykorgan, Almaty district, Kazakhstan \\ 3 I. Zhansugurov Zhetysu State University, Taldykorgan, Almaty district, Kazakhstan \\ 4 National Agricultural Research Education Centre, Astana, Kazakhstan \\ College of Science and Engineering, School of Biological Sciences, Flinders University of South Australia, Bedford Park, SA, Australia \\ Corresponding Author: Yuri Shavrukov \\ Email address: yuri.shavrukov@flinders.edu.au
}

Background. The pathogens from Fusarium species can cause Fusarium root rot (RR) and other diseases in plant species including sugar beet (Beta vulgaris L.), and they have a strong negative impact on sugar beet yield and quality.

Methods. Twenty-two sugar beet breeding lines were evaluated for the symptoms of RR after inoculation with Fusarium oxysporum Sch., isolate No. 5, and growth in a field trial. Two candidate genes for RR resistance, $B V S P 2$ and BVSE2, encoding chitinases Class IV and III, respectively, were previously identified in sugar beet, and used for genotyping using modern Amplifluor-like SNP genotyping approach. The qPCR expression analysis was used to verify responses of the candidate genes for root rot infections.

Results. A strong association of two SNP markers for BVSP2 and BVSE2 with resistance to RR in sugar beet was found in our study. Very high BVSP2 expression (hundred-fold compared to Controls) was observed in three RR resistant accessions (2182, 2236 and KWS2320) 14 days after inoculation which returned to the control level on Day 18. RR sensitive breeding line 2210 showed a delay in mRNA level, reaching maximal expression of BVSP2 18 days after inoculation. The gene BVSE2, showed a strong expression level in leaf samples from the infected field trial only in the breeding line 2236, which showed symptoms of RR, and this may be a response to other strains of $F$. oxysporum. 
1 Two sugar beet chitinase genes, BvSP2 and BvSE2, analysed with SNP

2 Amplifluor-like markers, are highly expressed after Fusarium root rot 3 inoculations and field susceptibility trial

6 Raushan Yerzhebayeva ${ }^{1}$, Alfiya Abekova ${ }^{1}$, Kerimkul Konysbekov ${ }^{2}$, Sholpan Bastaubayeva ${ }^{1}$, 7 Aynur Kabdrakhmanova ${ }^{3}$, Aiman Absattarova ${ }^{1,4}$, Yuri Shavrukov ${ }^{5 *}$

1 - Kazakh Research Institute of Agriculture and Plant Production, Almalybak, 040909 Almaty 11 district, Kazakhstan;

$122^{2}$ - Taldykorgan branch, Kazakh Research Institute of Agriculture and Plant Production, 13 Taldykorgan, Almaty district, Kazakhstan;

143 - I. Zhansugurov Zhetysu State University, Taldykorgan, Almaty district, Kazakhstan;

154 -Current address: National Agricultural Research Education Centre, Astana, Kazakhstan;

165 - College of Science and Engineering, School of Biological Sciences, Flinders University, 17 Bedford Park, SA, Australia;

* - Corresponding Author. Yuri Shavrukov

E-mail: yuri.shavrukov@flinders.edu.au 


\section{Abstract}

23 Background. The pathogens from Fusarium species can cause Fusarium root rot (RR) and other 24 diseases in plant species including sugar beet (Beta vulgaris L.), and they have a strong negative 25 impact on sugar beet yield and quality.

26 Methods. Twenty-two sugar beet breeding lines were evaluated for the symptoms of RR after 27 inoculation with Fusarium oxysporum Sch., isolate No. 5, and growth in a field trial. Two 28 candidate genes for RR resistance, $B v S P 2$ and $B v S E 2$, encoding chitinases Class IV and III, respectively, were previously identified in sugar beet, and used for genotyping using modern Amplifluor-like SNP genotyping approach. The qPCR expression analysis was used to verify responses of the candidate genes for root rot infections.

Results. A strong association of two SNP markers for $B v S P 2$ and $B v S E 2$ with resistance to RR in sugar beet was found in our study. Very high BvSP2 expression (hundred-fold compared to Controls) was observed in three RR resistant accessions (2182, 2236 and KWS2320) 14 days after inoculation which returned to the control level on Day 18. RR sensitive breeding line 2210 showed a delay in mRNA level, reaching maximal expression of $B v S P 218$ days after inoculation. The gene $B v S E 2$, showed a strong expression level in leaf samples from the infected field trial only in the breeding line 2236, which showed symptoms of RR, and this may be a response to other strains of $F$. oxysporum. 
45

46

47

\section{Introduction}

Root rot (RR) has a strong negative impact on many crops, including sugar beet (Beta vulgaris L.), where both the quantity and quality of production from farmers can be significantly reduced (Martin 2003; Heydari and Pessarakli 2010; Chehri 2011). The most important component to consider in the broad scale management of root rot is host plant resistance and disease severity, where a greater investment must be paid to comparing the immune response of the available sugar beet breeding lines and varieties to root rot pathogens (Eggestein et al. 2008; Christ and Varrelmann 2011).

Phytopathological studies have revealed that a number of Fusarium species can cause Fusarium RR and other diseases in sugar beet like storage rot (Lupashku and Mereniuc 2010; Christ and Varrelmann 2011; Liebe and Varrelmann 2016). Three species, F. xylarioides, F. camptoceras and $F$. solani, were the most virulent species causing Fusarium RR in sugar beet grown in Egypt (Abo-Elnaga and Amein 2011; Abd-El-Khaira et al. 2013), whereas two species, $F$. solani and $F$. culmorum, were reported as the casual agents of the disease in the UK (Jacobsen 2006). In the USA, F. oxysporum has been identified as a major cause of Fusarium RR in sugar beet (Harveson and Rush 1997, 1998; Jacobsen 2006; Christ and Varrelmann 2011), as well as in Moldova and in the south-eastern part of Kazakhstan (Maui 2002; Lupashku and Mereniuc 2010; Urazaliev et al. 2013).

Infections in the roots of sugar beet plants in the field are known to be caused by a complex of several phytopathogens in different countries (Jacobsen 2006; Liebe et al. 2016), including Kazakhstan, where three major strains of F. oxysporum Sch., No. 5, 50 and 150, were isolated (Maui 2002; Mombekova et al. 2013). Therefore, it is important to define $F$. oxysporum strains, which can be identified in field trials. To this end, artificial inoculations of young plants with a single Fusarium strain in the laboratory can be used to accurately estimate virulence as well as the resistance of sugar beet genotypes to the fungus (Harveson and Rush 1998). Finally, expression analysis of candidate genes must be carried out and compared with the symptoms of natural fungal infection in field tests and with symptoms in laboratory tests using inoculations of a single Fusarium strain. This can help to identify and verify possible candidate genes for 
Fusarium RR resistance and shed light on the different reactions of sugar beet plants to Fusarium infection (Larson et al. 2007).

F. oxysporum is part of a diverse fungal genus containing many species that include pathogenic or non-pathogenic, highly virulent or avirulent forms causing RR, wilt and other disease symptoms in various plant species (Mirkova and Karadjova 1994; Harveson and Rush 1997; Christ and Varrelmann 2011; Webb et al. 2012, 2013; Covey et al. 2014). The metabolites produced by $F$. oxysporum are so toxic that they have been successfully used as a biocontrol agent in sunflower seeds and seedlings against parasitic plants of the genus Orobanche (Amsellem et al. 2001; Dor et al. 2007; Fan et al. 2007). Nevertheless, it was reported that mango and oil palm plants have the capacity to suppress or reduce pathogen attack by inducing the synthesis of chitinase (Ebrahim et al. 2011; Rusli et al. 2015).

The activity of chitinase enzymes (EC 3.2.1.14) is correlated with pathogenic infection and, therefore, may play an important role in the plant defence mechanisms within root or leaf cells against various phytopathogens, like $F$. oxysporum. For example, in sugar beet, chitinases were shown to be highly up-regulated in plants inoculated with $F$. oxysporum strain F-19, a fungus associated with the root disease Fusarium yellows (Larson et al. 2007). Similar results showing strongly increased chitinase production were reported in sugar beet leaves infected by Cercospora beticola (Nielsen et al. 1993, 1994), vascular wilt due to root infection by $F$. oxysporum in oil palm, Elaeis guineensis (Rusli et al. 2015), and resistance to floral malformation in mango, Mangifera indica L. (Ebrahim et al. 2011). Chitinases catalyse the hydrolysis of chitin, which is present in the cell walls of fungi and insects but is not found in plant cells (Collinge et al. 1993; Nagpure et al. 2014). Therefore, chitinases may be associated with tolerance to Fusarium RR in other crops including sugar beet plant species.

Chitinases fall into five classes, some of which are specific to plants only and others that are also present in bacteria and fungi (Roopavathi et al. 2015), and are produced by plants in response to infection by either a single or particular group of pathogens (Roopavathi et al. 2015). Sugar beet is plant species capable of producing chitinase in association with a response to pathogenic fungi (Nielsen et al. 1993, 1994; Larson et al. 2007). Chitinases have received significant attention due 
106 to their effectiveness in a wide range of applications, including their use as a biocontrol agent

107 against plant-pathogenic fungi (Nagpure et al. 2014).

108

109 Two isoforms of acid chitinase (SE1 and SE2) were identified in leaves of sugar beet in response

110 to infection by the leaf-spot fungus Cercospora beticola, but only one of the isoforms (SE2)

111 showed exochitinase activity and could effectively hydrolyse chito-oligosaccharides (Nielsen et

112 al. 1993). SE2 shows most similarity to a Class III chitinase in Arabidopsis, tobacco and

113 cucumber. The sugar beet SE2 gene (designated as BvSE2 = Beta vulgaris SE2) showed a much

114 higher level of expression after infection by Cercospora in tolerant compared to susceptible

115 cultivars (Nielsen et al. 1993). Application of a cell-wall protein solution extracted from the non-

116 pathogenic oomycete Pythium oligandrum isolate onto sugar beet leaves initiated a strong

117 defensive reaction. This involved the significant increase of BvSE2 gene expression, with

118 maximal value at four hours after inoculation (Takenaka and Tamagake 2009). However, no

119 chitin is present in oomycetes, so BvSE2 may be induced as part of a co-ordinated response to

120 other pathogenesis-related proteins (Collinge et al. 1993).

121

122 Similar to SE1 and SE2, two isoforms of another acid chitinase (SP1 and SP2) were also found 123 and described in leaves of sugar beet infected by C. beticola (Nielsen et al. 1994). Only one 124 glycosylated isoform, SP2, was characterised as a Class IV chitinase, similar to SP2 genes found 125 in rapeseed, bean and maize (Collinge et al. 1993). Expression of the BvSP2 (= Beta vulgaris 126 SP2) gene was very high in infected leaves of sugar beet plants, but BvSP2 protein accumulation 127 was limited to the area immediately surrounding the sites of infection (Nielsen et al. 1994). 128 Sugar beet chitinase IV gene BvSP2 when expressed in transgenic birch (Betula pendula) 129 inhibited growth of the insect larvae of Orgyia antiqua L., Lymantriidae (Vihervuori et al. 2013) 130 but had no adverse effects on mammalian herbivores like roe deer (Capreolus capreolus L.) 131 (Vihervuori et al. 2012).

133 Molecular markers, including SNP (Single nucleotide polymorphism), are currently employed as 134 routine method for the molecular genetic analysis of candidate genes. Amplifluor (Amplification 135 with fluorescence) SNP analysis is a novel technology for high-throughput, accurate SNP 136 genotyping that is based on a similar platform to KASP (Kompetitive allele specific PCR) 
137 markers (He et al. 2014). Amplifluor-like SNP markers have a simple design, employing a

138 mixture of two universal probes carrying fluorescent labels and a quencher, and non-labelled 139 gene-specific primers (Giancola et al. 2006; Shavrukov et al. 2016; Jatayev et al. 2017). Based 140 on our recent study in bread wheat (Shavrukov et al. 2016), we extended our research to sugar 141 beet via the development of primers targeting SNPs in an effort to identify candidate genes for 142 RR resistance.

143

144 The aims of this study were: (1) to evaluate a set of sugar beet breeding lines and accessions for 145 Fusarium RR resistance in comparative tests between artificial inoculation with $F$. oxysporum 146 Sch. in the laboratory and natural infections of an unidentified pathogen(s) in field trials; (2) to 147 identify SNPs for the design and scoring of SNP Amplifluor-like markers specific to the two 148 chitinase genes $B v S E 2$ and $B v S P 2$; and (3) to analyse gene expressions in response to inoculation 149 with F. oxysporum, strain No. 5, and infection in field trials.

\section{Materials and Methods}

\section{Plant material}

153 The twenty-two sugar beet breeding lines (accessions) used in this study are listed in 154 Supplementary Table S1 with their corresponding origins. All breeding material is self155 incompatible, with population-based selection and propagation employed for their production. 156 Seeds were supplied by the Taldykorgan branch of the Kazakh Research Institute of Agriculture 157 and Plant Production, Taldykorgan, Almaty district, Kazakhstan. Seeds of KWS2320 were 158 kindly provided by KWS SAAT SE, Einbeck, Germany. The genome of KWS2320 as the 159 Reference line has been completely sequenced (Dohm et al. 2014) and is publicly available on 160 the NCBI database (Accession No. PRJNA74567; www.ncbi.nlm.nih.gov).

Artificial inoculation with F. oxysporum fungal suspension in the laboratory and resistance 163 scoring of infected plants

164 For the laboratory test, sugar beet seedlings were grown in 10-cm diameter pots filled with field165 collected, autoclaved soil $\left(120^{\circ} \mathrm{C}, 20 \mathrm{~min}\right.$ treatment $)$ in a greenhouse at $28^{\circ} \mathrm{C} / 22^{\circ} \mathrm{C}$ with $16 \mathrm{~h} / 8 \mathrm{~h}$ day/night to the 3-4 leaf growth stage. Fungal inoculation was carried out as described by Walker (1969) with the following steps. Cuts were made with a sterile scalpel measuring one $\mathrm{cm}$ in 
168 length, penetrating approximately $1 / 3$ of the width in the top part of the root in the pot-grown 169 plants. F. oxysporum Sch., strain No. 5, was isolated earlier from infected roots in a previous 170 field trail among two other major strains No. 50 and 150 (Maui 2002). The isolates of $F$. 171 oxysporum were inoculated onto the surface of Czapek media and grown until they were 172 undergoing rapid growth, as described earlier (Thom and Church 1926). All types of conidia 173 were collected by scalpel and mixed into $1 \mathrm{ml}$ of sterile water. One drop $(50 \mu 1)$ of the resulting 174 suspension was added into the incision and allowed to absorb into the root tissue. The 175 inoculation was repeated 10 days later in the same razor cut and in the same plants. Fusarium RR 176 symptoms were assessed after one month (on leaf blades and petioles) and two months (in roots) 177 following the date of the first inoculation. In laboratory tests, 10 plants were scored in each 178 accession.

The field trials were conducted in the Taldykorgan, Almaty district, on south-eastern part of Kazakhstan during two years (2016 and 2017). Plants of each breeding line were grown in a 16 $\mathrm{m}$ row, with four rows in each plot, $60 \mathrm{~cm}$ between rows, $18-20 \mathrm{~cm}$ between plants, and a planting density of 5-6 plants within one metre of a row. Four replicates of plots in each breeding lines were completely randomised. Plants in the field trial received no artificial inoculations. Ten plants growing in the same general area were randomly selected for scoring in each replicate in the field trial; scoring was repeated for four replicate plots in each accession. The total number of scored biological replicates (plants) in field was 40 for each breeding line.

An identical five-point scoring system was applied in both the laboratory tests and the field trial, as follows: 0 - Highly resistant (disease development 0-15\% of plants); 1 - Stable (disease development 16-30\% of plants); 2 - Average resistance (disease development 31-50\% of plants); 3 - Susceptible (disease development 51-70\% of plants); 4 - Highly susceptible (disease development 71-100\% of plants) (Khovanskaya et al. 1985; Urazaliev et al. 2013). Each score was based on two assessments (leaf blades/petioles and roots) in the same inoculated plants in the laboratory test and in plants without inoculation in the field trial. If scores in both assessments were identical, it was recorded as a common result, but both scoring results were recorded in the form of a range, if the two assessment scores differed. Non-inoculated plants in 198 the laboratory test were used as controls with a $\mathbf{0}$ score. 
DNA extraction, bioinformatics and sequencing for SNP identification

201

202

203

204

205

206

207

208

209

210

211

212

213

214

215

216

217

218

219

220

221

222

223

224

225

226

227

228

229

DNA was extracted from young plants at the 5-6 leaf stage, using the CTAB method (Dellaporta et al. 1983). The concentration of DNA was measured using a NanoDrop (ThermoFisher, Germany) and was then adjusted with sterile water to $10 \mathrm{ng} / \mu \mathrm{l}$.

DNA sequences were obtained from the annotated line KWS2320 on the NCBI database (www.ncbi.nlm.nih.gov); BvSE2 from LOC104888158 and BvSP2 from LOC104888888. Primers were designed to cover entire genes, based on the annotated sequences. Sequences of $B v S E 2$ and $B v S P 2$, as well as primers for sequencing and gene-specific SNP markers KIZ4 and KIZ3, as well as the positions of SNPs identified in this study, are provided in Supplementary Figures S1 and S2, respectively. Amplified PCR products for BvSE2 and BvSP2 genes (1,076 and 1,609 bp, respectively), were purified using a PCR Purification kit (Qiagen, Australia) and Sanger sequenced from both 5'- and 3'-ends using a service in the Australian Genome Research Facility, Adelaide, Australia. The comparison of BvSE2 and BvSP2 sequences in breeding lines from Kazakhstan and KWS2320 was used to test for the presence of SNPs. Two independent biological replicates were used to verify the accuracy and stability of the sequence. The SNP positions identified were then used for primer design and SNP Amplifluor-like analysis.

\section{SNP Amplifluor-like genotyping assay}

Amplifluor-like SNP analysis, as described earlier (Shavrukov et al. 2016; Jatayev et al. 2017), was carried out using a QuantStudio-7 Real-Time PCR Cycler (ThermoFisher Scientific, USA). The PCR cocktail in each well contained 2xMaster-Mix with the following reagents in their final concentrations: $1.8 \mathrm{mM} \mathrm{MgCl}, 0.25 \mu \mathrm{M}$ each fluorescent label probe, $0.2 \mathrm{mM}$ each of dNTPs, $0.15 \mu \mathrm{M}$ of each forward primer, $0.78 \mu \mathrm{M}$ of reverse primer, $1 \times$ PCR Buffer and 0.5 units of Taq DNA polymerase (GenLab, Astana, Kazakhstan). Half of the total PCR volume (5 $\mu$ l out of 10 $\mu 1)$ was genomic DNA, adjusted for $10 \mathrm{ng} / \mu \mathrm{l}$, and $5.0 \mu \mathrm{l}$ or $2.5 \mu \mathrm{l}$ of each DNA sample was added in 96- or 384-well microplates, respectively. One $\mu \mathrm{l}$ of 1:100 diluted ROX was added as a passive reference label to the Master-Mix according to the manufacturer's protocol for the PCR Cycler. 
230 PCR cycling followed a program adjusted from those published earlier (Shavrukov et al. 2016;

231 Jatayev et al. 2017), and included initial denaturation at $95^{\circ} \mathrm{C}$ for $1 \mathrm{~min} ; 26-28$ cycles of $95^{\circ} \mathrm{C}$ for

$23230 \mathrm{~s}, 55^{\circ} \mathrm{C}$ for $30 \mathrm{~s}$, and $72^{\circ} \mathrm{C}$ for $50 \mathrm{~s}$; and final extension at $72^{\circ} \mathrm{C}$ for $5 \mathrm{~min}$. Genotyping with

233 SNP calling was determined automatically by software accompanying the instrument, but each

234 SNP result was checked manually using amplification curves and allele discrimination.

235 Genotyping experiments were repeated twice (two technical replicates) over different days, 236 where technical replicates confirmed the confidence of SNP calls.

238 Genotyping of eight individual plants in each breeding line was scored based on the majority of 239 the identified alleles in SNP analysis for both studied genes, $B v S P 2-a_{1} a_{1} ;-a_{1} b_{1} ;-b_{1} b_{1}$; and $240 B v S E 2-a_{2} a_{2} ;-a_{2} b_{2} ; b_{2} b_{2}$. The same plants were used for phenotyping scores, where all studied 241 breeding material was highly heterogeneous for both BvSE2 and BvSP2. Reference line 242 KWS2320 was homozygous for both genes, BvSP2- $a_{1} a_{1}$ and $B v S E 2-a_{2} a_{2}$.

RNA extraction, $c D N A$ construction and $q P C R$ analysis for gene expression

Plants were grown in the laboratory until 3-4 leaf growth stage, as described above. All leaves were collected from each plant immediately prior inoculation with $F$. oxysporum Sch., strain No. 5 (Day 0, used as Control), with following similar samplings of other plants from the same pots, 14 and 18 days after the inoculation, based on our preliminary tests. In the field trial, two spots were designated as non-infected (Controls, C), where plants had no symptoms of RR and others were designated as infected (I) areas where they showed severe RR symptoms after one month of growth. Two biological replicates for each treatment and control were used. RNA was extracted from leaf samples of individual plants both in the laboratory and in the field trial using TRIsollike reagents, as published earlier (Shavrukov et al. 2013). RNA quality was checked by agarose gel electrophoresis with following treatments for 15 minutes, at room temperature, with $1 \mu 1$ of DNase I (ThermoFisher, Dreieich, Germany) to remove any traces of DNA. cDNA was constructed from $2 \mu \mathrm{g}$ of DNase-treated RNA using oligo- $\mathrm{dT}_{(18)}$ and Reverse Transcriptase kit, M-MuLV-RH (Biolabmix, Novosibirsk, Russia; http://www.biolabmix.ru) following the manufacturer's instructions. 
260 Non-diluted cDNA samples were used for qPCR analyses in a Real-Time PCR system, Model 261 CFX96 (BioRad, Gladesville, NSW, Australia) at Flinders University, Australia. The total 262 volume of $10 \mu \mathrm{l}$ qPCR reactions included $5 \mu \mathrm{l}$ of 2 x KAPA SYBR FAST (KAPA Biosystems, $263 \mathrm{USA}), 4 \mu \mathrm{l}$ of cDNA, and $1 \mu \mathrm{l}$ of mixed forward and reverse gene-specific primers $(3 \mu \mathrm{M}$ of 264 each). Expression data for the target genes, BvSP2 and BvSE2, were normalized using the 265 reference gene, Glutamine synthetase (LOC104883503), as suggested and used for sugar beet 266 (Taski-Ajdukovic et al. 2012) and repeated twice (two technical replicates). It was checked with 267 infected and control plants as a suitable reference gene prior to the analysis. The Relative 268 standard curve method is based on the ABI Guide to performing relative quantitation of gene 269 expression using real-time quantitative

PCR (http://www3.appliedbiosystems.com/cms/groups/mcb_support/documents/generaldocuments/c ms_042380.pdf), where serial dilutions were used for each target and reference gene individually (Soole and Smith 2015). Based on linear calibration of template cDNA dilution factor and $\mathrm{Cq}$ value, the threshold cycle values were determined. The coefficients of determination, $\mathrm{R}^{2}$, were greater than 0.995 in all studied target and reference genes. The efficiencies of all qPCR primers were calculated based on slope of the corresponding calibration line, $E=10^{\text {slope }}$ (Borges et al. 2012), and they were in the suitable range, 1.8-2.0. Specificities of target and reference genes were confirmed by single distinct peaks on a melting curve and a single band of the expected size in 2\% agarose gel electrophoresis. Details of primer sequences and product sizes are present in Supplementary Table S2.

Statistical analysis

The non-parametric Mann-Whitney $U$-test was applied for ranking scores in pair comparisons, as suggested for plant disease severity (Shah and Madden 2004), and the online calculator was used at http://www.socscistatistics.com/tests/mannwhitney. The Mann-Whitney $U$-test in this case operates with Nominal variable or Categorical (ordinal) variable data (Harris et al. 2008); also with a small number of analyses in each studied accession (Fagerland 2012), making it the most suitable statistical treatment for ranking score comparisons in pairs. The paired Sign test was used to analyse if two sets of scores were correlated with each other using the online calculator http://www.socscistatistics.com/tests/signtest/Default.aspx. For gene expression analysis, 
290 average and standard errors were calculated using standard Excel software. Probabilities for

291 significance, $\mathrm{P}<0.05$ and $\mathrm{P}<0.01$, were calculated using Student's $t$-test.

292

293 Results

294

Structure of the chitinase genes BvSE2 and BvSP2, and their localisation on chromosome $3 S$

295

296

297

298

299

300

301

302

303

304

305

306

307

308

309

310

311

312

313

314

315

316

317

318

319

320

Sequences of $B v S E 2$ and $B v S P 2$ were annotated using sequence obtained from the sugar beet genome database (NCBI). The full sequence of sugar beet BvSE2 chitinase, Class III was identical in all annotated accessions (S66038, AAB28479, XM_010673039 and LOC104888158) including Acc. 567433940 for the doubled haploid (DH) line KWS2320 using a Whole genome shotgun sequencing approach (WGSGS). The ORF was 882 base pairs (bp) with 293 predicted amino acids (aa) making up the corresponding protein BvSE2 (Acc. XP_010671341). Similarly, sugar beet annotated accessions, L25826, AAA32916, XM_010673997 and LOC104888888 with Acc. 730225012 for WGSGS, were identified for the sequence of BvSP2 chitinase, Class IV. In the comparison of the two chitinases, both ORF of the BvSP2 gene and the BvSP2 protein sequence were shorter, comprising 867 bp and 288 aa, respectively (XP_010671341). No SNP or other polymorphism was found in the databases for either $B v S E 2$ or $B v S P 2$ genes.

The chromosome locations of $B v S E 2$ and $B V S P 2$ on sugar beet chromosome 3 were indicated in the annotated LOC accessions. The positions of these genes on the physical map of chromosome 3S were identified using published information with flanking SNP markers, EBS0085 and BB02714 (Holtgräve et al. 2014). Using WGSGS, the genes were located in the interval between 97,069 and 122,718 bp on the short arm of chromosome 3; the position of BVSE2 was determined as distal and close to SNP marker EBS0085, while BvSP2 was proximal and located slightly further from the SNP marker BB02714 (Fig. 1).

\section{[Insert Figure 1 here]}

Sequence analysis revealed SNPs in $\mathrm{BvSE} 2$ and $\mathrm{BvSP} 2$

Two sugar beet breeding lines (2217 and 2263), with different germplasm origin (Supplementary Table S1), were selected for sequencing. Two biological replicates were included for each accession. The sequenced genetic regions in the BvSE2 and BvSP2 genes comprised 1,076 and 
321 1,609 bp, respectively. The fragments encompass both 5'- and 3'-UTR, and cover entire genes,

322 including introns. Sequencing of $B v S E 2$ and $B v S P 2$ fragments from both ends of the DNA

323 fragments revealed the full sequence identity when compared to KWS2320. A single SNP was

324 identified in the BvSE2 and BvSP2 genes, with substitutions [W=A/T] and $[\mathrm{Y}=\mathrm{C} / \mathrm{T}]$ at

325 positions 377 and 345 from the Start-codon, in BvSE2 and BvSP2, respectively. Both identified

326 SNPs were heterozygotes in the two biological replicates studied. This means that one allele at

327 each SNP position was identical to the sequence in KWS2320 but the other differed. It is

328 important to note that the $\mathrm{SNP}[\mathrm{W}=\mathrm{A} / \mathrm{T}]$ corresponded to a substitution of $\mathrm{N}_{126}$ with $\mathrm{I}_{126}$ in the

329 predicted amino acid sequence of the BvSE2 protein, but the SNP $[\mathrm{Y}=\mathrm{C} / \mathrm{T}]$ of $\mathrm{R}_{115}$ was 330 synonymous.

331

332

333

334

335

336

337

338

339

340

341

342

343

344

345

346

347

348

349

350

351

Phenotyping of the response to F. oxysporum Sch. inoculation, strain No. 5, and genotyping with an SNP Amplifluor-like marker for BvSP2 chitinase gene, Class IV

Ten individual plants for each of twenty-two accessions plus KWS2320 were examined for response to F. oxysporum Sch., strain No. 5, as described in the Materials and Methods. Laboratory phenotype scores assessing the apparent resistance levels one and two months after inoculation were consistent. An overview of scoring results is presented in Table 1. Breeding lines 1017, 2282 and 2296 showed the highest resistance (score of 0-1) to F. oxysporum, strain No. 5, identical to those of KWS2320. More than half of all studied accessions were classified as resistant, with scores ranging between 1 and 2. Three lines had an equal number of resistant and susceptible plants. Two lines (2125 and 2210) after inoculation with $F$. oxysporum strain No. 5, showed the strongest symptoms of susceptibility to the phytopathogen (Table 1A).

\section{[Insert Table 1 here]}

Genotyping results were based on self-designed Amplifluor-like SNP markers, and amplification with either FAM or VIC produced fluorescence signals linked with the corresponding SNP. This quantitative fluorescence was determined by the qPCR instrument and used for allele discriminations with automatic SNP calls among individual plants in different breeding lines. Examples of allele discrimination for the SNP in $B v S P 2$ and $B v S E 2$ are presented in Figure 2. The genotypes formed clearly separate two groups with homozygotes and relatively small 
352 portion of heterozygote SNPs. All genotypes of KWS2320 plants fell into the homozygote 353 classes, $B v S P 2-a_{1} a_{1}$ and $B v S E 2-a_{2} a_{2}$.

354

355

[Insert Figure 2 here]

356

357 The genotyping results of plants used for Fusarium RR phenotyping, when tested with the SNP 358 Amplifluor-like marker KIZ3 for the BvSP2 gene, revealed similar but not identical results 359 (Table 1A). Only KWS2320 had identical genotyping scores for all studied plants, while the 360 majority of the genotypes, $B v S P 2-a_{1} a_{1} ;-a_{1} b_{1}$; and $-b_{1} b_{1}$, were estimated in the other breeding 361 lines. Genotyping results showed a high level of association (20 out of 22 lines in total) with the 362 phenotypic estimation of the resistance or susceptibility to $F$. oxysporum, strain No. 5 ( $\mathrm{P}<0.01$, 363 Sign test). Two cases of non-opposed mismatches for BvSP2 gene were found between 364 phenotyping and genotyping scores (in lines 1042 and 2154) with an intermediate phenotyping 365 score (2) and genotyping of $B v S P 2-a_{1} a_{1}$ alleles (Table 1A).

366

367

Comparison of inoculation and field trial scores with genotyping using an SNP Amplifluor-like 368 marker for the BvSE2 chitinase gene, Class III

A comparison of disease severity ratings to Fusarium RR in sugar beet accessions using inoculation with $F$. oxysporum isolate, strain No. 5, and to RR in plants grown in a field trial is presented in Table 1A and 1B. Most of the breeding lines (16 in the laboratory test and 14 in the

372 field trial) out of 22 studied were resistant to root rot. However, a number of accessions were classified as susceptible (score ratings 3 or 4) and differed in the phenotyping score categories, accounting for two and five lines in the laboratory test and field trial, respectively.

Results of the comparison between scores for the laboratory (Table 1A) and field tests (Table 1B) revealed that more than half (12 out of 22) of the studied breeding lines showed identical or very similar score results. Seven lines had overlapping RR scores, with a mostly intermediate phenotype between resistant and susceptible or a mixture of both. This indicates a high degree of similarity between symptom scores of RR in the two different tests. Finally, three conflicting cases (Breeding lines 1002, 2210 and 2236) were found with opposing RR scores in the laboratory test and the field trial (Table 1, indicated in Bold). 
384 Genotyping results using the SNP Amplifluor-like marker KIZ4 for $B v S E 2$ gene, $B v S E 2-a_{2} a_{2}$; 385 $a_{2} b_{2}$; and $b_{2} b_{2}$, showed a strong association with the RR scores in plants grown in the field trial

386

387

388

389

390

391

392

393

394

395

396

397

398

399

400

401

402

403

404

405

406

407

408

409

410

411

412

413 [Insert Figure 3 here] (Table 1B). Four out of five breeding lines susceptible to RR in the field trial $(1042,2154,2236$ and 2262) had a perfect match with the genotyping results. The remaining accessions susceptible to RR (1002 for field trial, and 2172 and 2263 for $B v S E 2-a_{2} b_{2}$ genotypes) showed intermediate scores in both the field trial and genotyping. Eleven breeding lines were classified as recording very similar RR scores; all other lines had overlapping RR scores. No conflicting results with opposing RR scores for phenotyping in the field trial and genotyping with SNP marker for BvSE2 were found. KWS2023 was resistant to RR in both laboratory inoculation and in the field trial (Table 1).

Based on results presented in Table 1, three breeding lines with contrasting reactions to inoculation with $F$. oxysporum, strain 5 in the laboratory test and RR score in field trials, were selected for gene expression analysis. Line 2182 was identified as the most resistant in both tests; line 2210 was sensitive to strain 5 inoculation but resistant to RR in field trials; and, in contrast, line 2236 was resistant to strain 5 inoculation but sensitive to RR in field trials. There was no identified breeding line sensitive in both laboratory and field tests. KWS was used as comprehensive reference line.

\section{Gene expression using $q P C R$}

Expression analysis of $B v S P 2$ and $B v S E 2$ genes after inoculation with $F$. oxysporum, strain 5 , showed that three selected lines (2182, 2236 and KWS2320) had very high expression on Day 14 after inoculation, which returned to levels similar to Controls at Day 18. In contrast, in RR sensitive breeding line 2210, the expression of $B v S P 2$ was 4-7.5-fold smaller at Day 14 compared to three RR resistance lines but it was 200-fold higher on Day 18 (Fig. 3A). In leaf samples from the infected field trial, only accession 2236 showed significantly increased expression of BvSE2 compared to other samples from infected field trials as well as to all Controls (Fig. 3B). 
415 Discussion

416 Reduced yields and crop quality due to root rot in sugar beet can be resolved through the 417 introduction of resistant genotypes (Jacobsen 2006; Christ and Varrelmann 2011), a goal that is 418 especially important for the region of Kazakhstan. Genetic polymorphism for resistance and 419 susceptibility to Fusarium species causing Fusarium RR has been reported for infected Egyptian 420 sugar beet cultivars in field trials (Abd-El-Khaira et al. 2013), and our own results presented 421 herein provide similar findings for genetic diversity among studied sugar beet germplasms in 422 Kazakhstan. In our experiments, only three breeding lines, 1017, 2282 and 2296, remained free 423 of RR symptoms in the laboratory test, and three other breeding lines, 2182, 2210 and 2261, 424 recorded no symptoms in the field trial. Most breeding lines segregated for RR resistance in the 425 laboratory test, field trial or in both. This observation can be directly related to the high level of 426 heterogeneity resulting from general self-incompatibility in the species Beta vulgaris L. (Kalia 427 2005), which is typical in sugar beet material derived from population-based selection 428 (Archimowitsch 1956). Nevertheless, the strong association (Sign test, $\mathrm{P}<0.01$ ) was found in our 429 study between Fusarium RR in laboratory and SNP genotyping for BvSP2 from one side (Table 430 1A), and between RR symptoms in field trials and SNP genotyping for BvSE2 from another side 431 (Table 1B). This can indicate that both studied chitinase genes, BvSP2 and BvSE2, are very likely 432 differentially involved in the reaction of plants to infection with Fusarium species. All 433 mismatching cases between genotyping and phenotyping in Table 1 are 'overlapped' with 434 intermediate scores, and they can be explained by the probable high level of heterogeneity in the 435 studied breeding lines.

436

In Moldova and Southern Kazakhstan, F. oxysporum was previously determined (Lupashku and Mereniuc 2010; Urazaliev et al. 2013) to be a cause of Fusarium RR in sugar beet, where three major strains of F. oxysporum Sch., No. 5, 50 and 150, were isolated (Maui 2002). Nevertheless, more precise taxonomic and molecular analyses are required to reliably distinguish $F$. oxysporum

441 from other reported forms of the fungus in sugar beet (Harveson and Rush 1998; Jacobsen 2006; 442 Christ and Varrelmann 2011). 
444 The fungal strains or species involved in causing Fusarium RR can vary considerably depending 445 on geographic location, soil conditions, planting time, crop rotations in the field, herbicide 446 applications and other factors (Jacobsen 2006; Lupashku and Mereniuc 2010). This means that 447 cultivars or hybrids resistant to Fusarium RR in one area can be more or less susceptible in other 448 areas. This may have contributed to the findings presented in the current study, where three 449 breeding lines (1002, 2210 and 2236) recorded conflicting RR scores in the field trial versus the 450 inoculation by $F$. oxysporum, strain No. 5, in the laboratory. This indicates the presence of an 451 additional factor influencing RR scores, very likely strains No. 50 or 150 of $F$. oxysporum, found

452

453 454 455

456 457 458 459 460 461 462 463 464 465 466 467 468 469 earlier in the same field trial (Maui 2002). Selected individuals from these accessions showing evidence of apparent resistance to Fusarium RR pathogens in one test and susceptibility in another should be carefully propagated and their seeds stored in Genebank.

Of greater interest to sugar beet breeders are obviously genotypes showing symptoms of resistance to all (or at least to several) Fusarium RR pathogens. Based on the current study, we can identify only two breeding lines, 2181 and 2282, which have shown resistance to both $F$. oxysporum strain No. 5 and to RR infection in the field, with scores ranging between 0 and 1. It is also important to point out the genetic significance of genotypes showing a very high susceptibility to the studied RR pathogen, but these genotypes might be unsuitable for the growing region of Kazakhstan.

Very high expression levels of $B v S P 2$ gene in leaves of sugar beet plants after inoculation strongly support the hypothesis that this candidate gene is responsive to $F$. oxysporum, strain 5 (Fig. 3A). It is shown very clearly, and with very high significance $(\mathrm{P}<0.01)$, that four days earlier (Day 14), the expression is associated with better resistance to the RR inoculation. This means that resistance to RR is very likely determined by a signalling system connecting with a defence mechanism, and this process is initiated by rapid and very strong expression of BvSP2 in leaves (and perhaps other parts of plants) in response to inoculation by $F$. oxysporum, strain 5 . In contrast, a delay in $B v S P 2$ expression response can slow down the defence mechanism and finally result in the sensitivity to RR, as seen in the breeding line 2210 (Fig. 3A). 
474 Different time points were used in leaf samplings for analysis of $B v S P 2$ and $B v S E 2$ gene 475 expression: 14 and 18 days after inoculation in the laboratory test and one month in the field trial 476 test, respectively. Changes in BvSP2 expression indicated a quicker response to the inoculation 477 in resistant lines 2282, 2236 and KWS2320 (Fig 3A) with maximum expression at Day 14. This 478 type of expression profile was very similar to those for BvSE2 after one month of growth in the 479 field trial, where the significant changes of BvSE2 gene expression were observed only in leaf 480 samples of accession 2236 collected from infected field trials (Fig. 3B). The expression of BvSE2 481 was very specific and occurred only in this single line, which displayed severe symptoms of RR 482 in the field test. Three RR resistant lines 2182, 2210 and KWS2320 typically show earlier 483 responses in $B v S E 2$ expression in response to root rot infections in the field, but BvSE2 484 expression levels returned back to initial levels after one month of plant growth in the infected 485 486 487 field trials. However, this hypothesis is hard to assess in field conditions where the timing of natural infections is not controlled and can be verified only by inoculation tests in the laboratory with known strains of $F$. oxysporum.

It is important to point out that no significant changes were registered in the expression of $B v S P 2$ 490 in the field test, which could indicate that strain 5 of $F$. oxysporum is not a dominant strain in 491 field conditions or that perhaps the natural activity and aggressiveness of this strain in soil was much reduced compared to artificial inoculation in the laboratory test. Therefore, we can speculate that the BvSE2 gene may be responsive to strains No. 50 or 150 of $F$. oxysporum, which were discovered and described earlier in the same field trials (Maui 2002).

At this stage, we can conclude that both candidate genes, $B v S P 2$ and $B v S E 2$, demonstrated differential expression responses after artificial inoculation and natural infection in soil, which is likely due to the differing compositions of $F$. oxysporum strains causing RR. The final step in the research would be to study the signalling system and protein-protein interactions between the pathogen and host plant to complete the story on how $F$. oxysporum causes Fusarium RR and how resistance or susceptibility arises in sugar beet plants in our experiments. Nevertheless, results presented in the current study indicate that both chitinase genes, $B v S P 2$ and $B v S E 2$, are strongly expressed in the plant in response to Fusarium RR. Interestingly, their roles appear to 504 differ depending on which strain of $F$. oxysporum is present, or possibly in response to 
505 interactions with environmental factors or other pathogenic species; an observation that requires 506 further investigation. This wider and more complex view of the mechanisms generating 507 resistance to Fusarium oxysporum isolate in sugar beet must be resolved in future studies.

508

\section{Conclusion}

510 A strong association of two SNP markers for $B v S P 2$ and $B v S E 2$ with resistance to RR in sugar 511 beet was found in our study. Very high BvSP2 expression (hundred-fold compared to Controls) 512 was observed in three RR resistant breeding lines (2182, 2236 and KWS2320) 14 days after 513 inoculation with Fusarium oxysporum, strain 5, and it was return to the control level on Day 18.

514 RR sensitive breeding line 2210 showed a delay in mRNA level, reaching maximal expression of $515 B v S P 218$ days after inoculation. The gene BvSE2, showed a strong expression level in leaf 516 samples of one month-old plants from the infected field trial only in the breeding line 2236, 517 which showed symptoms of RR, and this may be a response to other strains of F. oxysporum. 518 
520 References

521 Abd-El-Khaira H, Abd-El-Fattahb AI, El-Nagdic WMA (2013) Evaluation of five sugar beet

522 varieties for root-knot nematode and root-rot fungal infection. Arch Phytopathol Pflanzenschutz $523 \quad 46: 2163-2173$

524

525 Abo-Elnaga HIG, Amein KA (2011) Differentiation in protein patterns in Fusarium sp. causing 526 root rot and damping off diseases in sugar beet and wheat and their relation to pathogencity. Aust 527 J Basic Appl Sci 5:683-692

528

529 Amsellem Z, Barghouthi S, Cohen B, Goldwasser Y, Gressel J, Hornok L Kerenyi Z, Kleifeld Y, 530 Klein O, Kroschel J, Sauerborn J, Müller-Stöver D, Thomas H, Vurro M, Zonno MC (2001)

531 Recent advances in the biocontrol of Orobanche (broomrape) species. BioControl 46:211-228

532

533 Archimowitsch A (1956) Selective breeding of sugar-beets in Russia and the U.S.S.R. Bot Rev $53422(1): 1-37$

535

536 Christ D, Varrelmann M (2011) Fusarium in sugarbeet. Sugar Industry (Zuckerindustrie) 537 136:161-171 (In German)

538

539 Chehri K (2011) Occurrence of Fusarium species associated with economically important 540 agricultural crops in Iran. Afr J Microbiol Res 5:4043-4048

541

542 Collinge DB, Kragh KM, Mikkelsen JD, Nielsen KK, Rasmussen U, Vad K (1993) Plant 543 chitinases. Plant J 3:31-40

544

545 Covey PA, Kuwitzky B, Hanson M, Webb KM (2014) Multilocus analysis using putative fungal 546 effectors to describe a population of Fusarium oxysporum from sugar beet. Phytopathol 104:886547896

548

549 Dellaporta SL, Wood J, Hicks JB (1983) A plant DNA minipreparation: Version II. Plant Mol 550 Biol Rep 1:19-21 
551

552 Dohm JC, Minoche AE, Holtgräwe D, Capella-Gutiérrez S, Zakrzewski F, Tafer H, Rupp O, 553 Sörensen TR, Stracke R, Reinhardt R, Goesmann A, Kraft T, Schulz B, Stadler PF, Schmidt T, 554 Gabaldón T, Lehrach H, Weisshaar B, Himmelbauer H (2014) The genome of the recently 555 domesticated crop plant sugar beet (Beta vulgaris). Nature 505:546-549

556

557 Dor E, Evidente A, Amalfitano C, Agrelli D, Hershenhorn J (2007) The influence of growth 558 conditions on biomass, toxins and pathogenicity of Fusarium oxysporum f. sp. orthoceras, a 559 potential agent for broomrape biocontrol. Weed Res 47:345-352

560

561 Ebrahim S, Usha K, Singh B (2011) Pathogenesis-related (PR)-proteins: Chitinase and $\beta$-1,3562 glucanase in defense mechanism against malformation in mango (Mangifera indica L.). Sci 563 Hortic 130:847-852

564

565 Eggestein S, Grazhdankin A, Ugarov A (2008) Focus on the competitive edge of the sugar beet 566 industry - viewed through the prism of a plant breeding company. Int Sugar J 110(1312):222567228

568

569

Fagerland MW (2012) T-tests, non-parametric tests, and large studies paradox of statistical 570 practice? BMC Med Res Methodol 12. doi:10.1186/1471-2288-12-78

571

572 Fan ZW, Buschmann H, Müller-Stöver D, Sauerborn J (2007) Main effects and interactions 573 among acibenzolar-S-methyl, a biocontrol fungus and sunflower cultivar on control of 574 Orobanche cumana Wallr. J Plant Dis Prot 114:76-81

575

576 Giancola S, McKhann HI, Bérard A, Camilleri C, Durand S, Libeau P, Roux F, Reboud X, Gut 577 IG, Brunel D (2006) Utilization of the three high-throughput SNP genotyping methods, the 578 GOOD assay, Amplifluor and TaqMan, in diploid and polyploid plants. Theor Appl Genet 579 112:1115-1124 
581 Harris JE, Boushey C, Bruemmer B, Archer SL (208) Publishing Nutrition Research: A Review

582 of Nonparametric Methods, Part 3. J Amer Dietetic Assoc 108(9):1488-1496

583

584 Harveson RM, Rush CM (1997) Genetic variation among Fusarium oxysporum isolates from 585 sugar beet as determined by vegetative compatibility. Plant Dis 81:85-88

586

587 Harveson RM, Rush CM (1998) Characterization of Fusarium root rot isolates from sugar beet

588 by growth and virulence at different temperatures and irrigation regimes. Plant Dis 82:1039-1042 589

590

He C, Holme J, Anthony J (2014) SNP genotyping: the KASP assay. In: Fleury D, Whitford R 591 (eds) Crop Breeding: Methods and Protocols. Vol. 1145. Methods in Molecular Biology, 592 Springer, New York, pp 75-86

593

594 Heydari A, Pessarakli M (2010) A review on biological control of fungal plant pathogens using 595 microbial antagonists. J Biol Sci 10:273-290

596

597 Holtgräwe D, Sörensen TR, Viehöver P, Schneider J, Schulz B, Borchardt D, Kraft T, 598 Himmelbauer H, Weisshaar B (2014) Reliable in silico identification of sequence 599 polymorphisms and their application for extending the genetic map of sugar beet (Beta vulgaris). 600 PLoS One 9:e110113

601

602

Jacobsen BJ (2006) Root rot diseases of sugar beet. Proceed Natl Sci. Matica Srpska Novi Sad 603 110:9-19

604

605

Jatayev S, Kurishbayev A, Zotova L, Khasanova G, Serikbay D, Zhubatkanov A, Botayeva M, 606 Zhumalin A, Turbekova A, Soole K, Langridge P, Shavrukov Y (2017) Advantages of 607 Amplifluor-like SNP markers over KASP in plant genotyping. BMC Plant Biol 17(S2):254. doi:

608 $10.1186 / \mathrm{s} 12870-017-1197-\mathrm{x}$

609

610 Kalia P (2005) Root vegetable crops. J New Seeds 6:247-275

611 
612 Khovanskaya KN, Fradkina DL, Kazachenko RF, Khelman LV, Petrichenko SN, Peretyatko VG 613 (1985) Methodological instructions for a preparation of infection background and scores of sugar 614 beet cultivars for resistance to main diseases. VNIS, Kiev (in Russian)

615

616 Larson RL, Hill AL, Nuñez A (2007) Characterization of protein changes associated with sugar 617 beet (Beta vulgaris) resistance and susceptibility to Fusarium oxysporum. J Agric Food Chem $618 \quad 55: 7905-7915$

619

620 Liebe S, Varrelmann M (2016) Effect of environment and sugar beet genotype on root rot 621 development and pathogen profile during storage. Phytopathol 106:65-75

622

623 Liebe S, Christ DS, Ehricht R, Varrelmann M (2016) Development of a DNA microarray-based 624 assay for the detection of sugar beet root rot pathogens. Phytopathol 106:76-86

625

626 Lupashku GA, Mereniuc GV (2010) The influence of crop rotation and fertilizers on root rots 627 species composition and sugar beet sensitivity to them. Mycol Phytopathol 44:255-261 (in 628 Russian)

629

630

Martin HL (2003) Management of soilborne diseases of beetroot in Australia: A review. Aust J 631 Exp Agric 43:1281-1292

632

63

Maui AA (2002) Fungi from genera Fusarium Sch causing root rot in sugar beet. Bull Kazakh 634 Natl Univ Biol Ser 17(2):97-99 (In Russian)

635

636 Mirkova E, Karadjova Y (1994) New species of Fusarium in glasshouse carnations in Bulgaria. 637 Biotechnol Biotechnol Equip 8:8-12

638

639 Mombekova GA, Shemshura ON., Seitbattalova AI, Aitkhozhina NA, Bekmakhanova NE 640 (1993) Phytopathogens of sugar beet and soy cultivated in soil and climatic conditions of Almaty 641 region. Bull Natl Acad Sci Rep Kazakhtan. http://library.kz/en/menuvse/117642 journalsnanrk/vestnik/349-2013-4.html (In Russian) 
644 Nagpure A, Choudhary B, Gupta RK (2014) Chitinases: in agriculture and human healthcare.

645 Crit Rev Biotechnol 34:215-232

646

647 Nielsen KK, Mikkelsen JD, Kragh KM, Bojsen K (1993) An acidic class III chitinase in sugar 648 beet: induction by Cercospora beticola, characterization, and expression in transgenic tobacco 649 plants. Mol Plant Microbe Interact 6:495-506

650

651 Nielsen KK, Bojsen K, Roepstorff P, Mikkelsen JD (1994) A hydroxyproline-containing class 652 IV chitinase of sugar beet is glycosylated with xylose. Plant Mol Biol 25:241-257

653

654 Roopavathi AS, Vigneshwari R, Jayapradha R (2015) Chitinase: Production and applications. J 655 Chem Pharm Res 7:924-931

656

657 Rusli MH, Idris AS, Cooper RM (2015) Evaluation of Malaysian oil palm progenies for 658 susceptibility, resistance or tolerance to Fusarium oxysporum f. sp. elaeidis and defence-related 659 gene expression in roots. Plant Pathol 64:638-647

660

661 Shah DA, Madden LV (2004) Nonparametric analysis of ordinal data in designed factorial 662 experiments. Phytopathol 94:33-43

663

664 Shavrukov Y, Zhumalin A, Serikbay D, Botayeva M, Otemisova A, Absattarova A, Sereda G, 665 Sereda S, Shvidchenko V, Turbekova A, Jatayev S, Lopato S, Soole K, Langridge P (2016) 666 Expression level of the DREB2-type gene, identified with Amplifluor SNP markers, correlates 667 with performance and tolerance to dehydration in bread wheat cultivars from Northern 668 Kazakhstan. Front Plant Sci 7:1736. doi:10.3389/fpls.2016.01736

669

670 Soole K, Smith C (2015) Analysis of type II NAD(P)H dehydrogenases. In: Whelan J, Murcha 671 MW (eds) Plant Mitochondria: Methods and Protocols. Vol. 1305. Methods in Molecular 672 Biology, Springer, New York, pp 151-164

673 
674 Takenaka S, Tamagake H (2009) Foliar spray of a cell wall protein fraction from the biocontrol 675 agent Pythium oligandrum induces defence-related genes and increases resistance against 676 Cercospora leaf spot in sugar beet. J Gen Plant Pathol 75:340-348

677

678 Taski-Ajdukovic K, Nagl N, Kovacev L, Curcic Z, Danojevic D (2012) Development and 679 application of qRT-PCR for sugar beet gene expression analysis in response to in vitro induced 680 water deficit. Electron J Biotechnol 15(6). doi: 10.2225/vol15-issue6-fulltext-9

681

682 Thom C, Church MB (1926) The Aspergilli. Chapter IV. Culture of Aspergilli. The Williams and 683 Wilkins Company, Baltimore, pp 39-46

684

685

Urazaliev K, Abekova A, Bazylova T, Bersimbaeva G, Daniyarova A, Massonichich-Shotunova 686

R (2013) Somaclonal variation of sugar beet resistant to pathogenic root rot Fusarium 687 oxysporum var. orthoceras. Genetika 45:629-640

688

689

690

Vihervuori L, Lyytikäinen-Saarenmaa P, Tuomikoski E, Luoma M, Niemelä P, Pappinen A, Pasonen HL (2012) Palatability of transgenic birch and aspen to roe deer and mountain hare. 691 Biocontrol Sci Technol 22:1167-1180

692

693

Vihervuori L, Lyytikäinen-Saarenmaa P, Lu J, Pasonen HL (2013) Effects on lepidopteran 694 herbivores of feeding on leaves of transgenic birch (Betula pendula) expressing the sugar beet 695 chitinase IV gene. Eur J Entomol 110:253-262

696

Walker JC (1969) Plant Pathology, 3rd edn. McGraw-Hill Book, New York

698

Webb KM, Covey PA, Hanson LE (2012) Pathogenic and phylogenetic analysis of Fusarium 700 oxysporum from sugarbeet in Michigan and Minnesota. J Sugar Beet Res 49:38-56

701

702

Webb KM, Case AJ, Brick MA, Otto K, Schwartz HF (2013) Cross pathogenicity and vegetative 703 compatibility of Fusarium oxysporum isolated from sugar beet. Plant Dis 97:1200-1206 


\section{Table capture}

707 Table 1. Comparison of disease scores for Fusarium oxysporum Sch., isolate No. 5 symptoms in

708 sugar beet breeding lines, showing resistant, $\mathbf{R}(0$ or 1$)$, intermediate, In (2) and susceptible, $\mathbf{S}$ (3

709 or 4) scores, identified after inoculation in the laboratory (A) and growth in a field trial (B).

710 Genotyping of $B v S P 2$ and $B v S E 2$ was made according to the majority of identified alleles,

$711 \quad B v S P 2-a_{1} a_{1} ;-a_{1} b_{1} ;-b_{1} b_{1} ;$ and $B v S E 2-a_{2} a_{2} ;-a_{2} b_{2} ; b_{2} b_{2}$.

712 Mixed phenotyping scores of 1-2 or 2-3 in the laboratory and field trial were designated as resistant and susceptible 713 genotypes, respectively. The number of plants in the laboratory test and field trial were $n=10$ and $n=40$,

714 respectively, while $(n=8)$ plants were used for genotyping. All experiments were conducted twice. Significant 715 differences between pairwise comparisons according to the Mann-Whitney $U$-test $(P<0.05)$ are shown in bold and 716 marked with an asterisk (*). Other data showed no significant differences in pairwise comparisons using the same $717 U$-test. 
720 Figure legends:

721 Figure 1. Positions of $B v S E 2$ and $B v S P 2$ genes, encoding chitinases, Classes III and IV,

722 respectively, on the physical map of chromosome 3S in sugar beet. SNP markers were published

723 by Holtgräve et al. (2014), and LOC sequences were identified from the NCBI database

724

725 Figure 2. Allelic discrimination of SNP Amplifluor-like markers KIZ3 and KIZ4, designed for 726 the $B v S P 2$ and $B v S E 2$ genes encoding chitinases, Class IV and Class III, respectively, among 727 sugar beet breeding lines. Red and blue dots indicate automatic SNP calls for homozygotes in 728 Allele $1\left(B v S P 2-a_{1} a_{1} ; B v S E 2-a_{2} a_{2}\right)$ and Allele $2\left(B v S P 2-b_{1} b ; B v S E 2-b_{2} b_{2}\right)$, respectively, while 729 green dots indicate heterozygotes $\left(B v S P 2-a_{1} b_{1} ; B v S E 2-a_{2} b_{2}\right)$ or mixed genotypes. Black squares 730 show NTC (No Template Control). X- and Y-axes show Relative amplification units, $\Delta$ Rn, for 731 FAM and VIC fluorescence signals, respectively, as determined by the qPCR instrument.

732

733 Figure 3. Quantitative expression analyses (qPCR) of $B v S P 2$ and $B v S E 2$ genes (clear and black

734 bars, respectively) in leaves of sugar beet plants. (A) Plants at the 3-4 leaf stage were inoculated 735 by Fusarium oxysporum Sch., isolate No. 5, and indicated Day 0, as Controls, for comparison with Days 14 and 18 since inoculations. (B) Mature plants from non-infected Controls (C), and

737 Infected (I) parts of field trails. Names of the four selected sugar beet lines are shown on the top 738 of each Figure panel. Expression data were normalised using the reference gene, Glutamine 739 synthetase (LOC104883503). Bars show the average for two biological and two technical 740 replicates in each measurement \pm Standard Error. Different letters indicate significant differences 741 with other samples at the same time-points and Controls $(\mathrm{P}<0.01$ for panel $\mathrm{A}$ and $\mathrm{P}<0.05$ for 742 panel B). 


\section{Supplementary material}

744 Supplementary Table S1. List and origins of the 22 breeding lines and the reference DH line 745 KWS2320.

746 Supplementary Figure S1. Sequence of the BvSE2 gene, encoding chitinase, Class III, 747 annotated for the line KWS2320.

748 Supplementary Figure S2. Sequence of the BvSP2 gene, encoding chitinase, Class IV, 749 annotated for the line KWS2320.

750 Supplementary Table S2. Primers used for qPCR and their amplification products. 
Figure 1

Positions of BVSE2 and BVSP2 genes, encoding chitinases, Classes III and IV, respectively, on the physical map of chromosome $3 S$ in sugar beet.

SNP markers were published by Holtgräve et al. (2014), and LOC sequences were identified from the NCBI database.

*Note: Auto Gamma Correction was used for the image. This only affects the reviewing manuscript. See original source image if needed for review.

\section{Chromosome 3S}

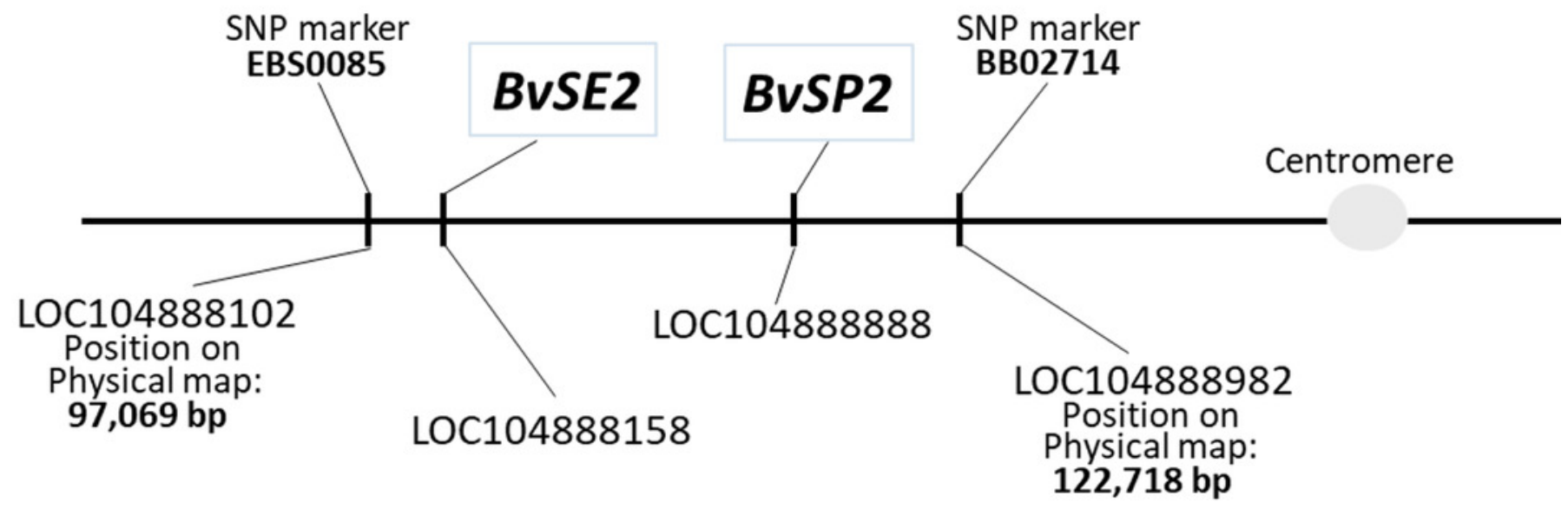




\section{Figure 2}

Allelic discrimination of SNP Amplifluor-like markers KIZ3 and KIZ4, designed for the BVSP2 and BVSE2 genes encoding chitinases, Class IV and Class III, respectively, among sugar beet breeding lines.

Red and blue dots indicate automatic SNP calls for homozygotes in Allele 1 (BVSP2-a $\left.a_{1} a_{1} ; B V S E 2-a_{2} a_{2}\right)$ and Allele $2\left(B V S P 2-b_{1} b ; B V S E 2-b_{2} b_{2}\right)$, respectively, while green dots indicate heterozygotes (BVSP2- $a_{1} b_{1}$; $B V S E 2-a_{2} b_{2}$ ) or mixed genotypes. Black squares show NTC (No Template Control). X-and Y-axes show Relative amplification units, $\triangle \mathrm{Rn}$, for FAM and VIC fluorescence signals, respectively, as determined by the qPCR instrument.
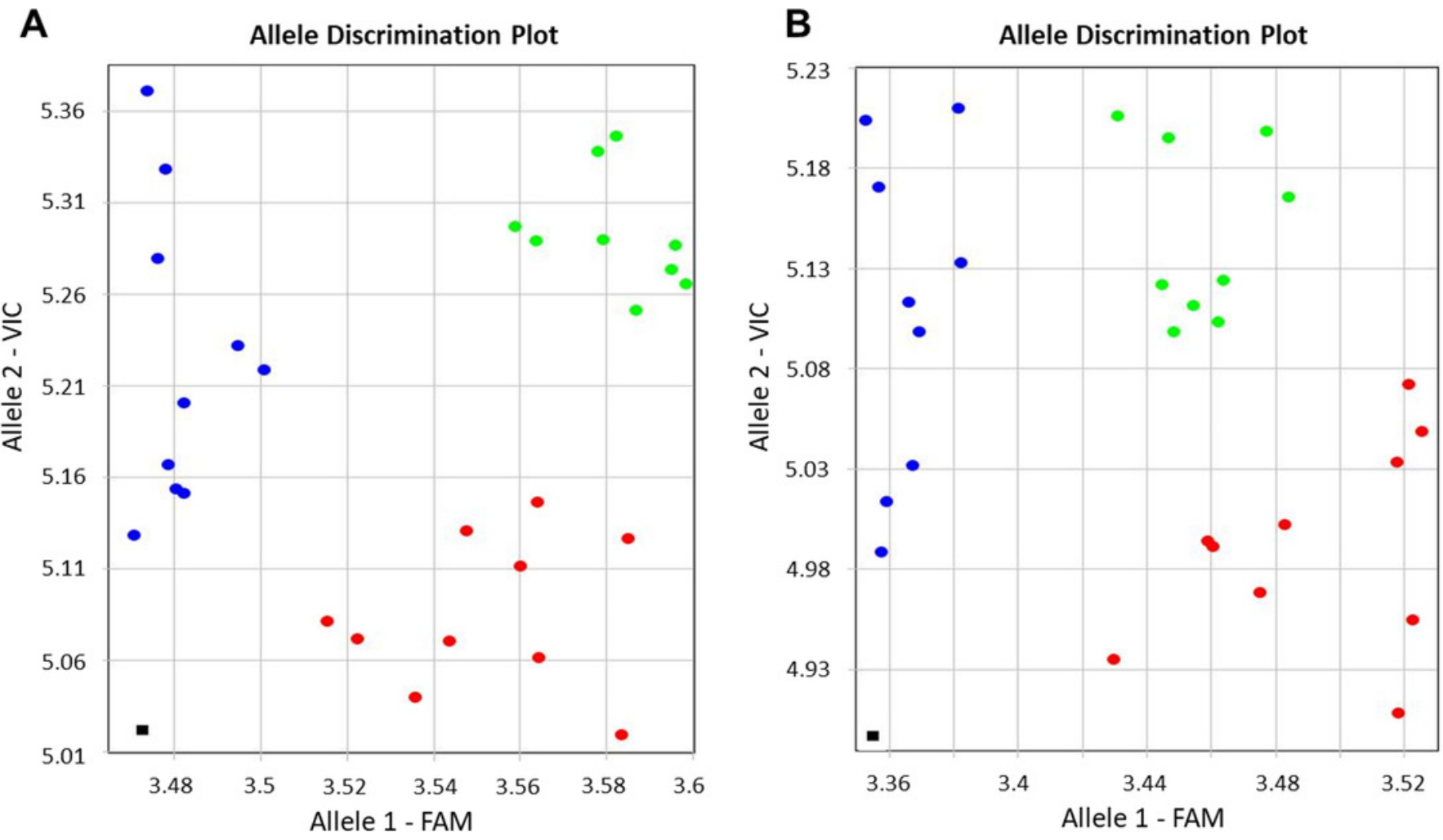


\section{Figure 3}

Quantitative expression analyses (qPCR) of BVSP2 and BVSE2 genes (clear and black bars, respectively) in leaves of sugar beet plants.

(A) Plants at the 3-4 leaf stage were inoculated by Fusarium oxysporum Sch., isolate No. 5, and indicated Day 0 , as Controls, for comparison with Days 14 and 18 since inoculations. (B) Mature plants from noninfected Controls (C), and Infected (I) parts of field trails. Names of the four selected sugar beet lines are shown on the top of each Figure panel. Expression data were normalised using the reference gene, Glutamine synthetase (LOC104883503). Bars show the average for two biological and two technical replicates in each measurement \pm Standard Error. Different letters indicate significant differences with other samples at the same time-points and Controls ( $\mathrm{P}<0.01$ for panel $\mathrm{A}$ and $\mathrm{P}<0.05$ for panel $\mathrm{B}$ ). 

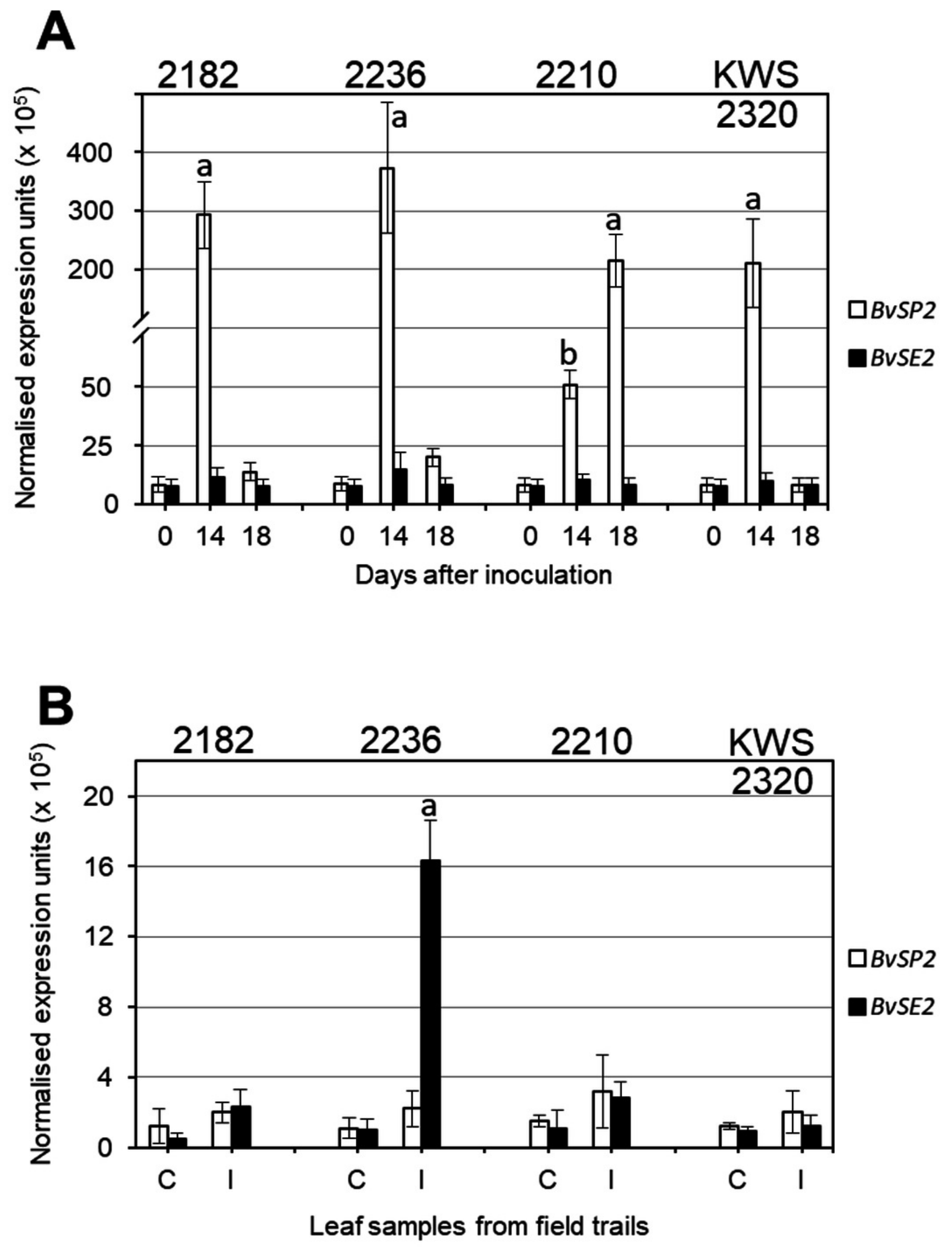


\section{Table $\mathbf{1}$ (on next page)}

Comparison of disease scores for Fusarium oxysporum Sch.

Comparison of disease scores for Fusarium oxysporum Sch., isolate No. 5 symptoms in sugar beet breeding lines, showing resistant, R (0 or 1), intermediate, In (2) and susceptible, S (3 or 4) scores, identified after inoculation in the laboratory (A) and growth in a field trial (B). Genotyping of BVSP2 and BVSE2 was made according to the majority of identified alleles, $B V S P 2-a_{1} a_{1} ;-a_{1} b_{1} ;-b_{1} b_{1} ;$ and BVSE2- $a_{2} a_{2} ;-a_{2} b_{2} ; b_{2} b_{2}$. 
1 Table 1. Comparison of disease scores for Fusarium oxysporum Sch., isolate No. 5 symptoms in 2 sugar beet breeding lines, showing resistant, $\mathbf{R}(0$ or 1$)$, intermediate, In (2) and susceptible, $\mathbf{S}$ (3 3 or 4 ) scores, identified after inoculation in the laboratory (A) and growth in a field trial (B). 4 Genotyping of $B v S P 2$ and $B v S E 2$ was made according to the majority of identified alleles, $5 \quad B v S P 2-a_{1} a_{1} ;-a_{1} b_{1} ;-b_{1} b_{1} ;$ and $B v S E 2-a_{2} a_{2} ;-a_{2} b_{2} ; b_{2} b_{2}$.

\begin{tabular}{|c|c|c|c|c|}
\hline \multirow{2}{*}{$\begin{array}{l}\text { Breeding } \\
\text { line ID }\end{array}$} & \multicolumn{2}{|c|}{ A } & \multicolumn{2}{|c|}{ B } \\
\hline & $\begin{array}{c}\text { Laboratory } \\
\text { score }\end{array}$ & $\begin{array}{c}\text { Genotyping of } \\
B v S P 2\end{array}$ & Field score & $\begin{array}{c}\text { Genotyping of } \\
B v S E 2\end{array}$ \\
\hline 1002 & $1 *, \mathrm{R}$ & $a_{1} a_{1}$ & $2-3 *, S$ & $a_{2} b_{2}$ \\
\hline 1005 & $1-2, \mathrm{R}$ & $a_{1} a_{1}$ & 2, In & $a_{2} b_{2}$ \\
\hline 1017 & $0-1, \mathrm{R}$ & $a_{1} a_{1}$ & $1-2, \mathrm{R}$ & $a_{2} b_{2}$ \\
\hline 1042 & 2, In & $a_{1} a_{1}$ & $2-3, \mathrm{~S}$ & $b_{2} b_{2}$ \\
\hline 1082 & $1-2, \mathrm{R}$ & $a_{1} a_{1}$ & $1, \mathrm{R}$ & $a_{2} a_{2}$ \\
\hline 2115 & $1-2, \mathrm{R}$ & $a_{1} a_{1}$ & $1, \mathrm{R}$ & $a_{2} a_{2}$ \\
\hline 2125 & $2-3, \mathrm{~S}$ & $b_{1} b_{1}$ & $1-2, \mathrm{R}$ & $a_{2} a_{2}$ \\
\hline 2154 & 2, In & $a_{1} a_{1}$ & $2-3, \mathrm{~S}$ & $b_{2} b_{2}$ \\
\hline 2172 & $1-2, \mathrm{R}$ & $a_{1} a_{1}$ & 2, In & $a_{2} b_{2}$ \\
\hline 2182 & $1, \mathrm{R}$ & $a_{1} a_{1}$ & $0-1, \mathrm{R}$ & $a_{2} a_{2}$ \\
\hline 2190 & $1-2, \mathrm{R}$ & $a_{1} a_{1}$ & $1-2, \mathrm{R}$ & $a_{2} a_{2}$ \\
\hline 2210 & $2-3 *, S$ & $b_{1} b_{1}$ & $0-1 *, \mathrm{R}$ & $a_{2} a_{2}$ \\
\hline 2217 & 2, In & $a_{1} b_{1}$ & $1-2, \mathrm{R}$ & $a_{2} b_{2}$ \\
\hline 2236 & $1-2 *, \mathrm{R}$ & $a_{1} a_{1}$ & $4 *, S$ & $b_{2} b_{2}$ \\
\hline 2261 & $1-2, \mathrm{R}$ & $a_{1} a_{1}$ & $0-1, \mathrm{R}$ & $a_{2} a_{2}$ \\
\hline 2262 & $1-2, \mathrm{R}$ & $a_{1} a_{1}$ & $2-3, \mathrm{~S}$ & $b_{2} b_{2}$ \\
\hline 2263 & $2, \mathrm{In}$ & $a_{1} b_{1}$ & 2, In & $a_{2} b_{2}$ \\
\hline 2281 & $1-2, \mathrm{R}$ & $a_{1} a_{1}$ & $1-2, \mathrm{R}$ & $a_{2} a_{2}$ \\
\hline 2282 & $0-1, \mathrm{R}$ & $a_{1} a_{1}$ & $1, \mathrm{R}$ & $a_{2} a_{2}$ \\
\hline 2286 & $1-2, \mathrm{R}$ & $a_{1} a_{1}$ & $1-2, \mathrm{R}$ & $a_{2} a_{2}$ \\
\hline 2296 & $0-1, \mathrm{R}$ & $a_{1} a_{1}$ & $1-2, \mathrm{R}$ & $a_{2} b_{2}$ \\
\hline 2300 & $1, \mathrm{R}$ & $a_{1} a_{1}$ & $1-2, \mathrm{R}$ & $a_{2} a_{2}$ \\
\hline KWS2320 & $0-1, \mathrm{R}$ & $a_{1} a_{1}$ & $0-1, \mathrm{R}$ & $a_{2} a_{2}$ \\
\hline
\end{tabular}

6 Mixed phenotyping scores of 1-2 or 2-3 in the laboratory and field trial were designated as resistant and susceptible 7 genotypes, respectively. The number of plants in the laboratory test and field trial were $n=10$ and $n=40$, 
8 respectively, while $(n=8)$ plants were used for genotyping. All experiments were conducted twice. Significant 9 differences between pairwise comparisons according to the Mann-Whitney $U$-test $(P<0.05)$ are shown in bold and 10 marked with an asterisk $(*)$. Other data showed no significant differences in pairwise comparisons using the same $11 U$-test.

12 